\title{
Research Paper: The Bay Area Functional Performance Evaluation-Task Oriented Assessment (BaFPE-TOA) in Severe Psychiatric Patients: A Psychometric Study
}

\author{
Ali Rashidian"1 , *Ashraf Karbalaei Nouri ${ }^{1} \odot$, Samaneh Hosseinzadeh², Hojjatollah Haghgoo ${ }^{1}$
}

1. Department of Occupational Therapy, University of Social Welfare and Rehabilitation Sciences, Tehran, Iran.

2. Department of Biostatistics, University of Social Welfare and Rehabilitation Sciences, Tehran, Iran.

\begin{tabular}{|c|c|}
\hline $\begin{array}{l}\text { Use your device to scan } \\
\text { and read the article online }\end{array}$ & detion: Rashidian A, Karbalaei Nouri A, Hosseinzadeh S, Haghgoo H. [The Bay Area Functional Performance Evaluation- \\
\hline 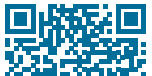 & $\begin{array}{l}\text { Task Oriented Assessment (BaFPE-TOA) in Severe Psychiatric Patients: A Psychometric Study (Persian)]. Archives of Rehabili- } \\
\text { tation. 2018; 19(2):168-177. http://dx.doi.org/10.32598/rj.19.2.168 }\end{array}$ \\
\hline retrist & doi: $:$ http://dx.doi.org/10.32598/rj.19.2.168 \\
\hline
\end{tabular}

Received: 09 Oct 2017

Accepted: 19 Mar 2018

\section{AB STRACT}

Objective The Bay Area Functional Performance Evaluation-Task Oriented Assessment (BaFPE-TOA) is a standard test developed by Williams and Bloomer in 1977-1978 in order to evaluate functional performance. This test consists of five tasks (sorting shells, money and marketing, home drawing, block design, kinetic person design), twelve parameters (memory for written and verbal instructions, organization, attention span, evidence of disorder, ability to abstract, completion, errors, efficiency, motivation and compliance, frustration tolerance, self-confidence, general affective impression) and three components (cognitive, affective, and performance components). The purpose of this study was to translate the BaFPE-TOA in Persian and to evaluate the face and content validity, and internal consistency in patients with severe psychiatric disorders.

Materials \& Methods A non-experimental and methodological study was carried out in Tehran in 2017; the study population included adult patients diagnosed with severe psychiatric disorders according to Diagnostic and Statistical Manual of Mental Disorders (DSM-5). In this study, demographic questionnaire and BaFPE test were used. Convenience Sampling was done on patients who were admitted to Razi Psychiatric Hospital. In order to prepare the Persian version, the translation of the BaFPE-TOA test was carried out in accordance with the International Quality of Life Assessment (IQOLA) project, which included: 1 . Translation of the original version into Persian; 2 . Review by translators and professors; 3. Translation quality; 4. Translation of the Persian version into English; and 5. Comparison of the English version with the original version. The Persian version was assigned to five experts to evaluate the content validity in terms of simplicity, relevance, clarity, and necessity. The face validity of the test was performed on three patients and two healthy individuals in order to find difficulties in the understanding of words and phrases, the appropriateness of the items, the likelihood of ambiguity and inadequate perceptions of expressions or the lack of meaning in words. After confirmation, a Persian version of the test was performed on 55 patients with severe psychiatric disorders who qualified the inclusion criteria. Inclusion criteria for this study included: 1 . People with psychiatric disorders based on DSM- 5 and are listed in the medical records by a psychiatrist; 2 . Over 18 years old; 3 . Complete consent to enter the study; 4 . Aability to write and read; 5 . Lack of comorbidity (mental retardation, drug abuse); 6 . Lack of physical constraints affecting the performance of specified tasks; 7 Lack of visual impairment or severe hearing loss. Cronbach's alpha coefficient was used to evaluate the internal consistency with respect to the four options of the test. Data analysis was performed using SPSS v.16 software.

Results Experts' opinions about the quality of translation and replacement of words in the translation process were according to the IQOLA method. The face validity of the test according to the experts' opinion and its association with healthy subjects and patients showed that the clarity and

\section{* Corresponding Author:}

Address: Department of Occupational Therapy, University of Social Welfare and Rehabilitation Sciences, Tehran, Iran. Tel: +98 (912) 8487250

E - Mail: nouri.uswr@gmail.com 
Keywords:

Psychiatric disorder, Occupational therapy, Assessment implementation of common language were of good quality. The content validity of the test was performed according to the opinion of the five experts in mental health; the Content Validity Ratio (CVR) and the Content Validity Index (CVI), were equal to one for all domains. The minimum acceptable value for content validity based on CVI and CVR indices was 0.80 and 0.99 , respectively, which indicated validity of the content and the lack of impact of culture on the parameters, tasks, and components of the BaFPE-TOA test. The results of the Cronbach's alpha coefficient for all fields was related to the twelve functional parameters, the three components, and the five tasks; the efficiency parameter was above 0.7 (the minimum acceptable value), indicative of the good internal reliability of this test.

Conclusion According to the experts' opinion, the Persian version of the BaFPE-TOA test has a proper translation, with acceptable face and content validity and internal consistency. Therefore, this test can be recommended for the evaluation of functional performance in patients with severe psychiatric disorders. 


\title{
مطالعه روانشناختى ابزار بررسى عملكرد كاركردى بِىاريا - ارزيابى مبتنى بر تكليف در بيماران شديد روانيزشكى روان مطنى
}

\author{
على رشيديان'، "اشرف كربلايى نورى' هـ سمانه حسينزاده'، حجتالله حق كو' \\ | 1 - كروه كاردرمانى، دائشكاه علوم بهزيستى و توانبخشى، تهران، ايران.

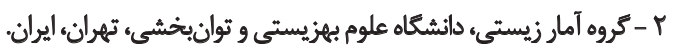

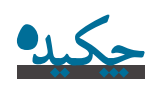

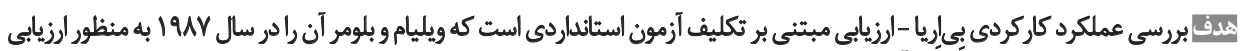

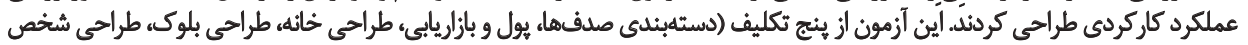

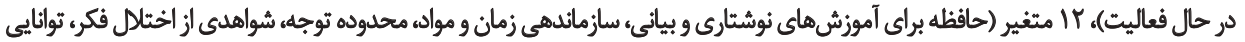

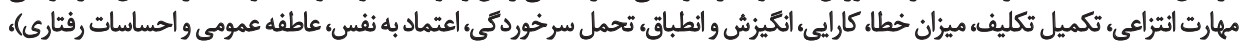

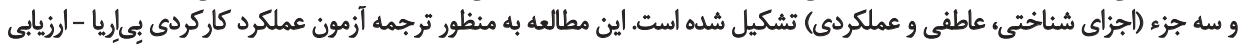

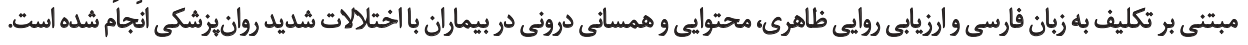

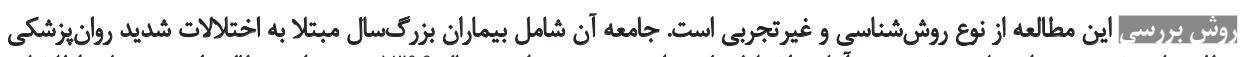

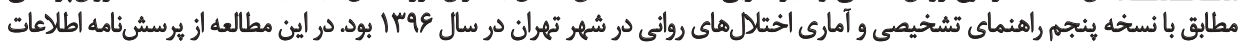

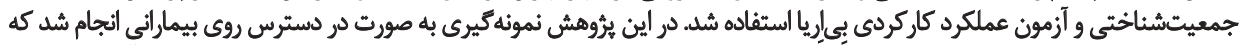

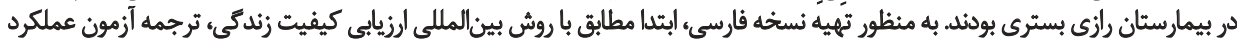

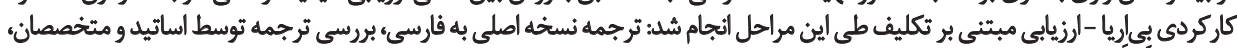

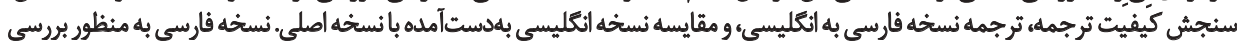

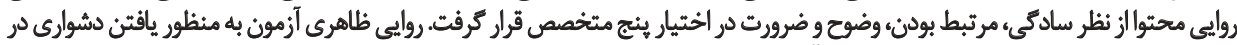

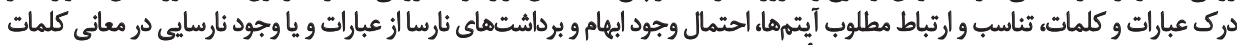

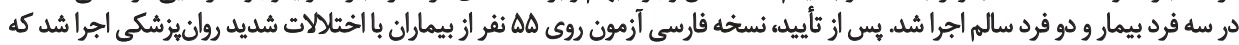

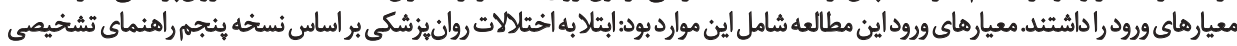

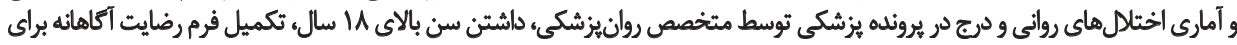

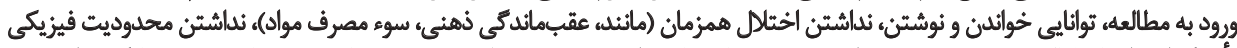

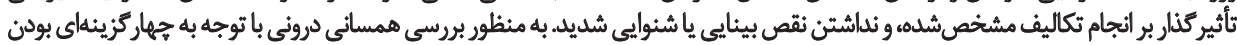

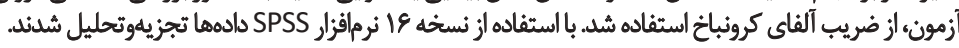

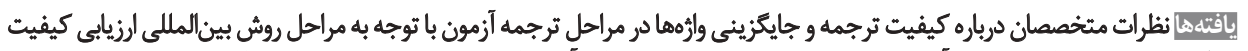

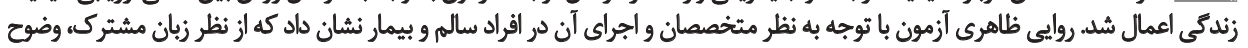

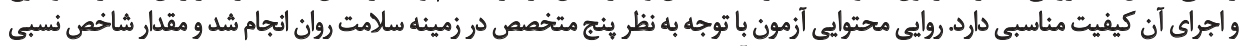

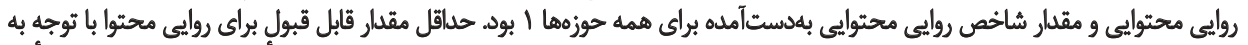

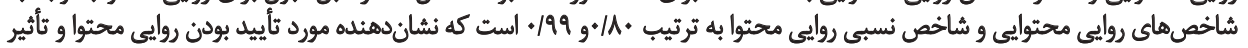

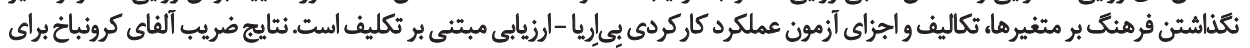

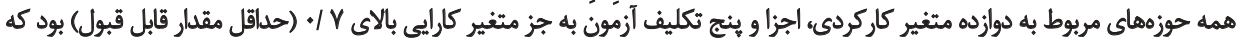

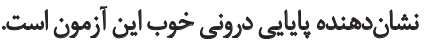

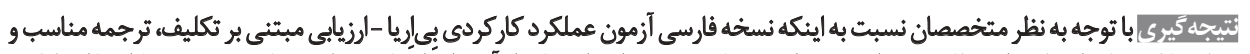

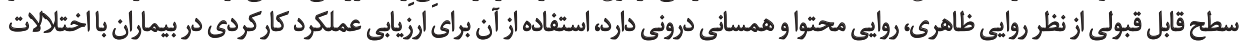
شديد روانيزشكى توصيه ميى أشود.
\end{abstract}

تاريخ دريافت: VI مهر 1499

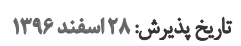

$$
\begin{aligned}
& \text { كليuدloglo } \\
& \text { بيماران روانيزئشكي، } \\
& \text { كاردرماني، ارزيابي }
\end{aligned}
$$

- 
در درمان، تعيين اولويت و برنامه درمانى و ميزان آمادكى براى

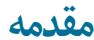

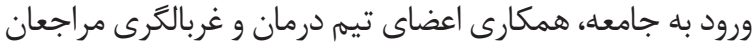
براى دريافت خدمات درمانى اشاره كرد [19، 19]. با توجه به اينكه نسخه فارسى و روايى و يايايى اين ابزار در داخل

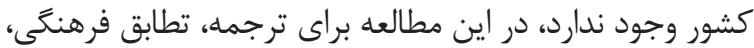

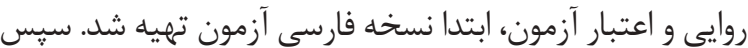

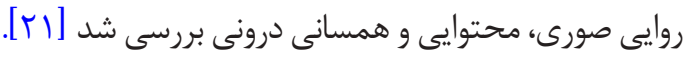

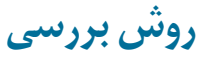

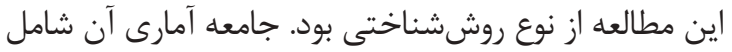

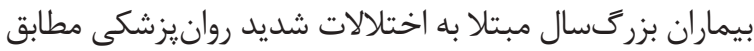

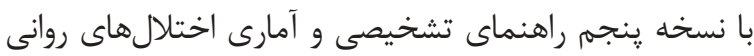

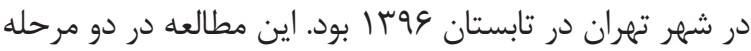

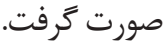

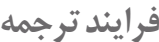

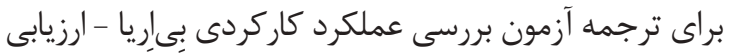

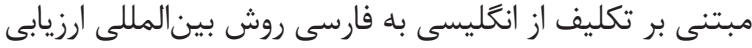

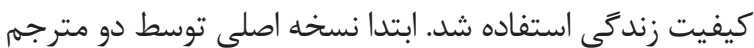

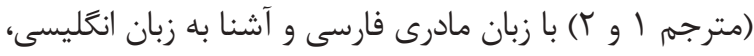

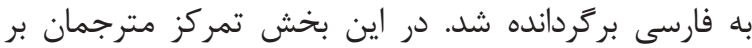

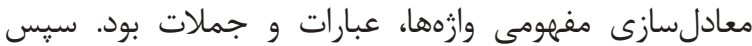

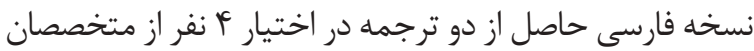

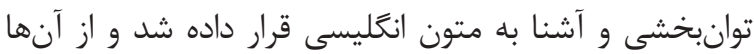

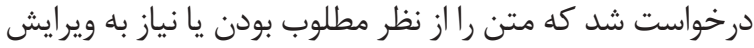

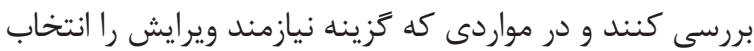

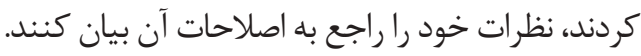

يس از اعمال ييشنهادات متخصصان، براى بررسى كيفيت

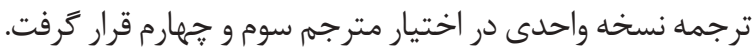

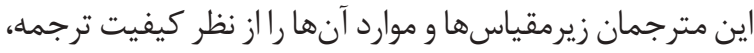

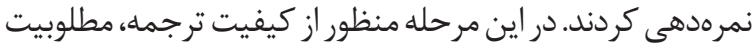

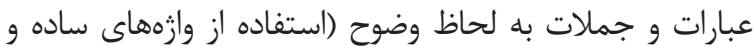

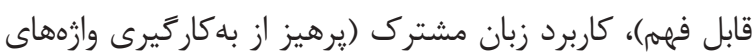

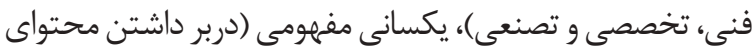

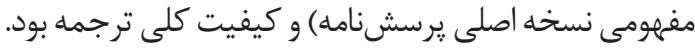
در اين مرحله نسخه فارسى تهيهشده در اختيار يك مترجمه

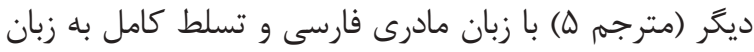

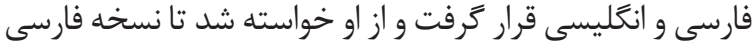

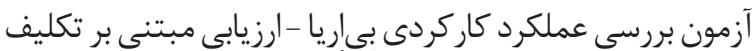

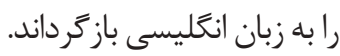

در نهايت نسخه انغليسى براى تأييد نهايى و اطمينان از يكسانى محتوايى نسخه فارسى و نسخه انغليسى اصلى ني در اختيار

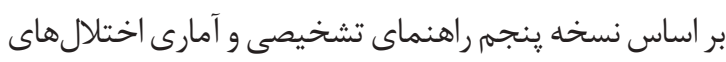

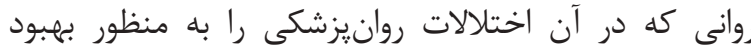

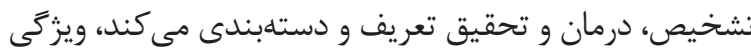

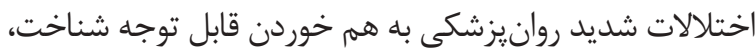

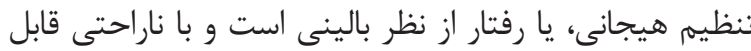

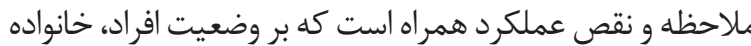

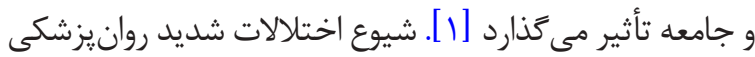

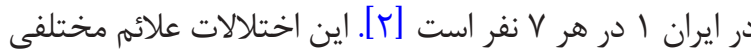

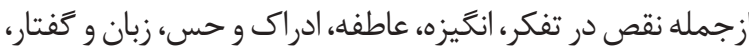

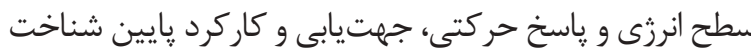

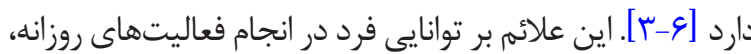

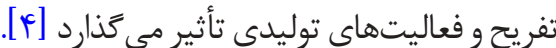
عملكرد كارى به توانايى افراد براى انجام فعاليتهاى روزمره

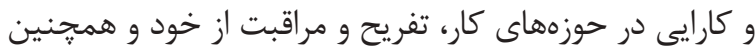

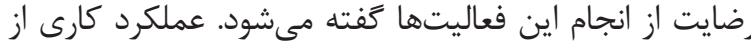

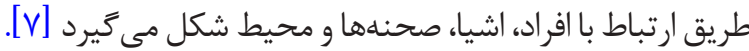

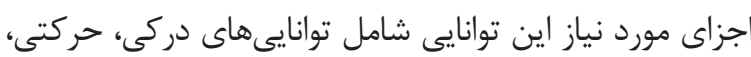

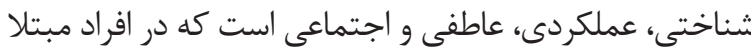

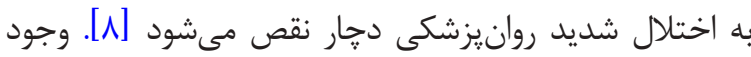

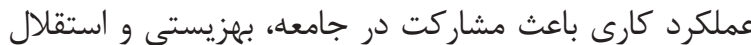

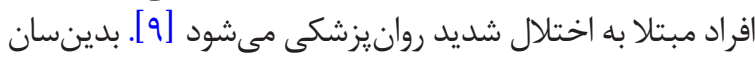

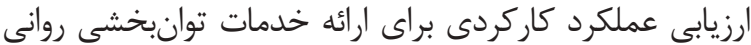

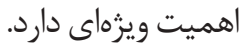

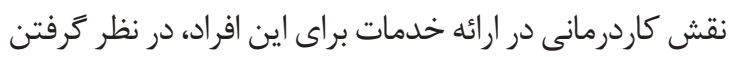

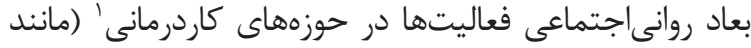

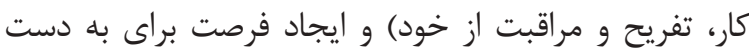

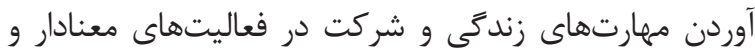

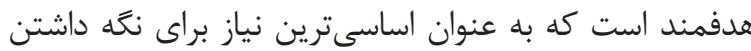

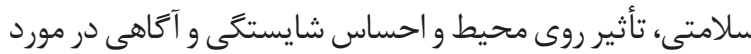

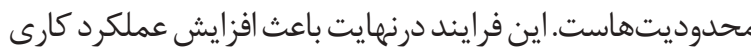

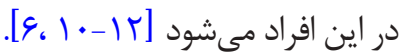

يكى از آزمونهاى معتبرى كه در كاردرمانى براى بررسى إردى إردي

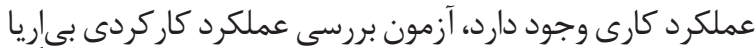

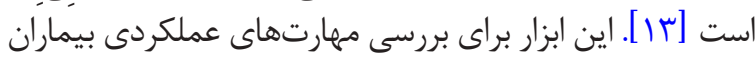

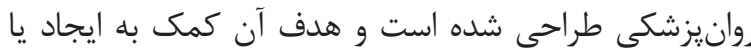

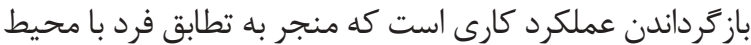

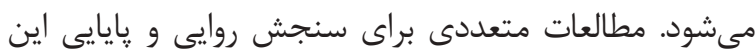

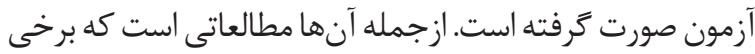

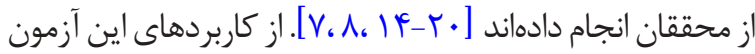

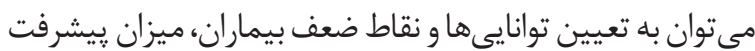

\section{Occupation}


آز مون بر رسى عملكرد كار كردى بِى إريا - ارزيابى مبتنى بر تكليف

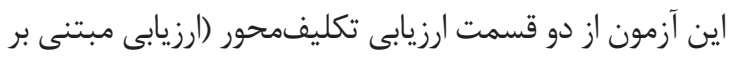

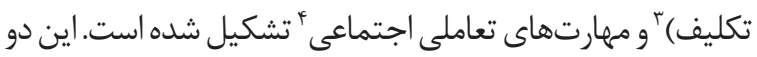

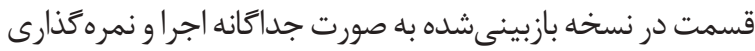

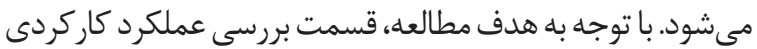

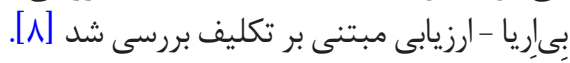

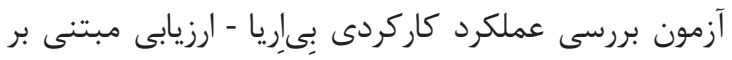

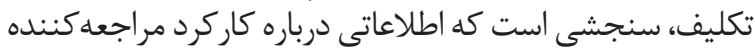

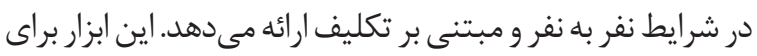

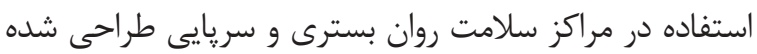

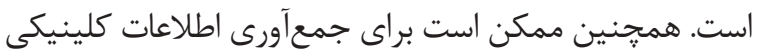

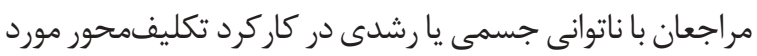

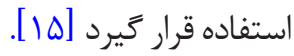

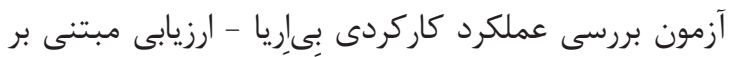

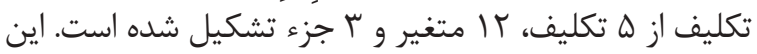
اجزا به شرح زير است: ت تكليف است ينج تكليف

تكليف دستابندى صدف ها: مراجعه كننده • ا نوع از صدفها

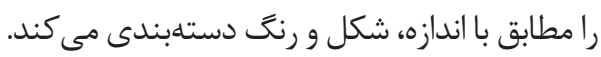
تكليف يول و بازاريابى: مراجعه كننده مقدار يول مورد نياز براى

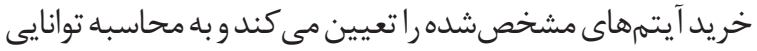

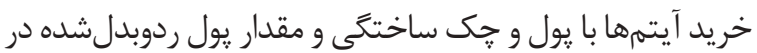
معامله مى يردازد.

تكليف طراحى خانه: مر اجعه كننده نقشه يك ساختمان را بعد إند

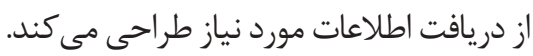

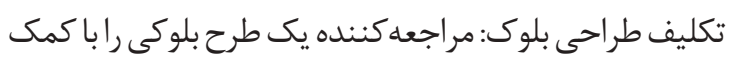

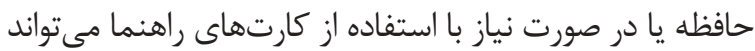

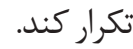

تكليف طراحى شخص در حال فعاليت: مراجعهكننده يك دام شخص را در حال انجام فعاليت نقاشى ميى كند.

$$
\text { دوازده متغير كاركردى }
$$

اجزاى شناختى: حافظه براى آموزشهاى نوشتارى و بيانى،

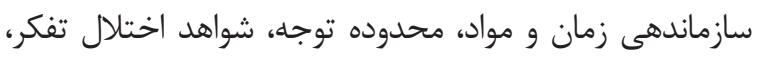
توانايى مهارت انتزاعى. اجزاى عملكردى: تكميل تكليف، ميزان خطا، كارايى.
فرد متخصص در زمينه توانبخشى و مسلط به زبان انخليسى

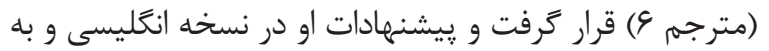

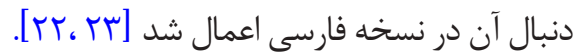

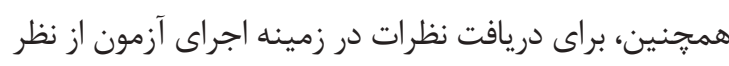

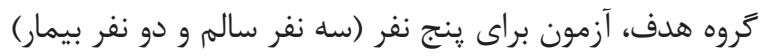

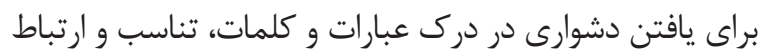

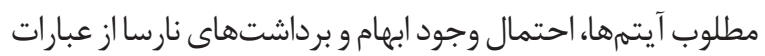

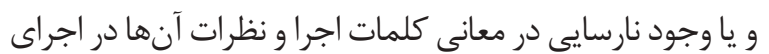

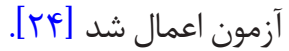

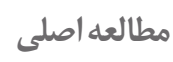

ابتدا از دانشكاه علوم بهزيستى و توانبخشى تأييديه تحقيق

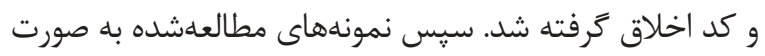

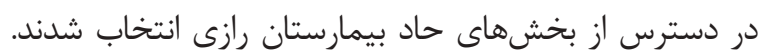

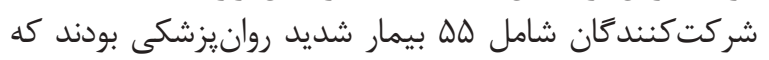

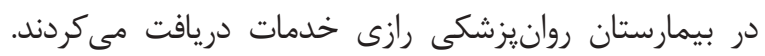

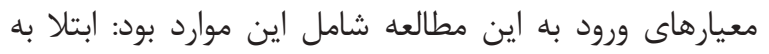

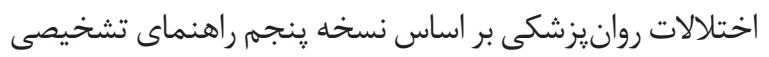

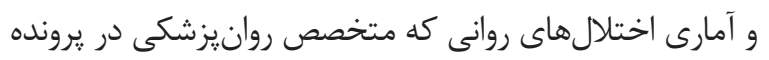

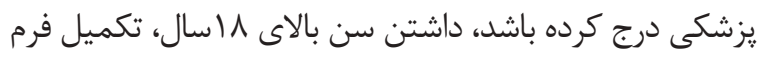

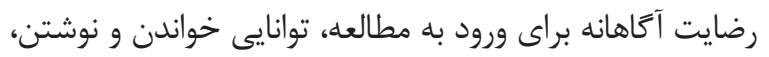

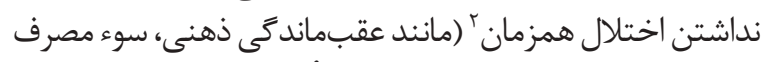

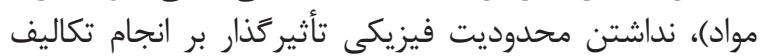

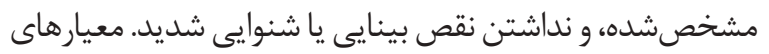

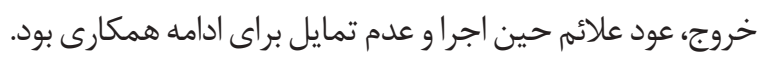

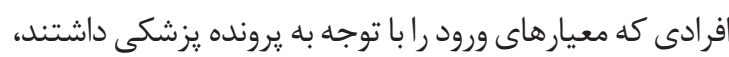

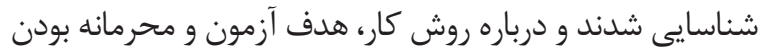

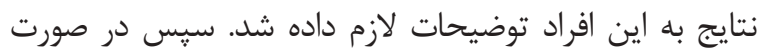

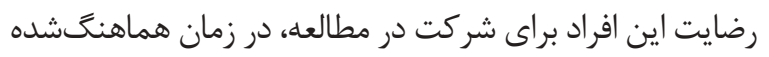
آزمونهاى لازم انجام شد.

ابزارها

در اين آزمون از يرسشنامه اطلاعات جمعيتشناختى و وردي

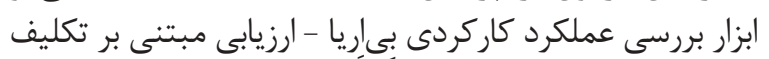

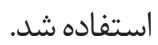

$$
\text { يرسش نامه اطلاعات فردى }
$$

اين يرسشنامه حاوى اطلاعات جمعيتشناختى شامل سطح

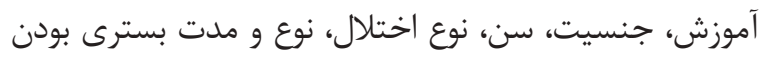

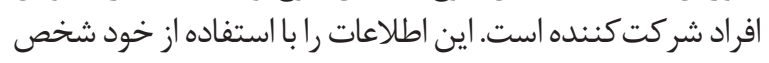
يا يرونده يزشكى به دست آمد آند. 


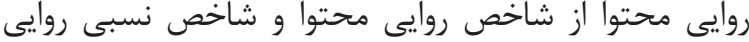

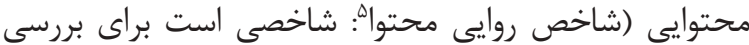

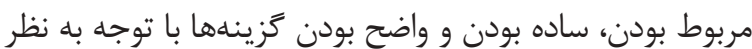

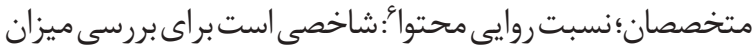

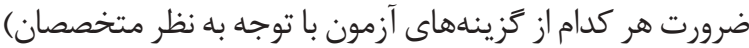

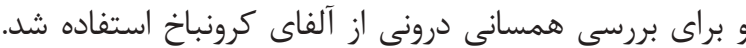

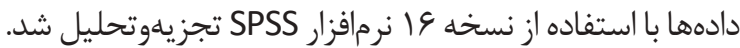

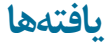

مشخصات جمعيتشناختى افراد شركت ركننده در جدول مبرول

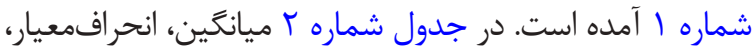

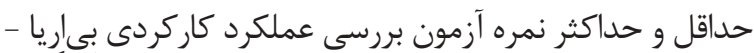

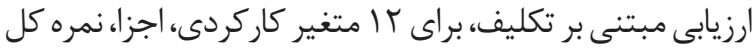

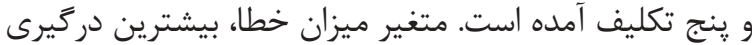

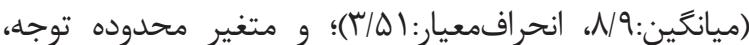

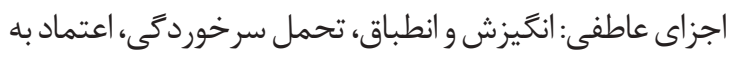
نفس، عاطفه عمومى و احساسات رفتارى [ه ا 1 ]. بركَهاى نمرهَذارى براى ثبت اطلاعات درباره كاركرد

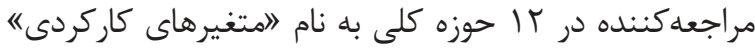

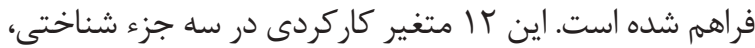

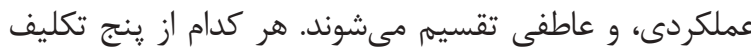

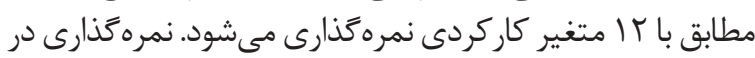

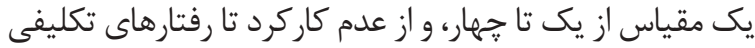

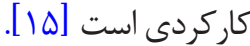

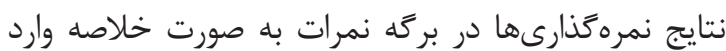

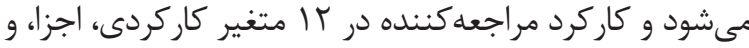

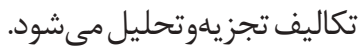

روش تجز يلهوتحليل دادهها

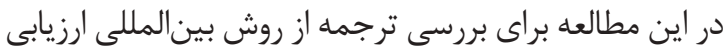

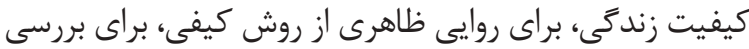

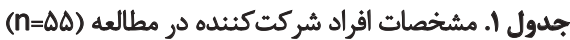

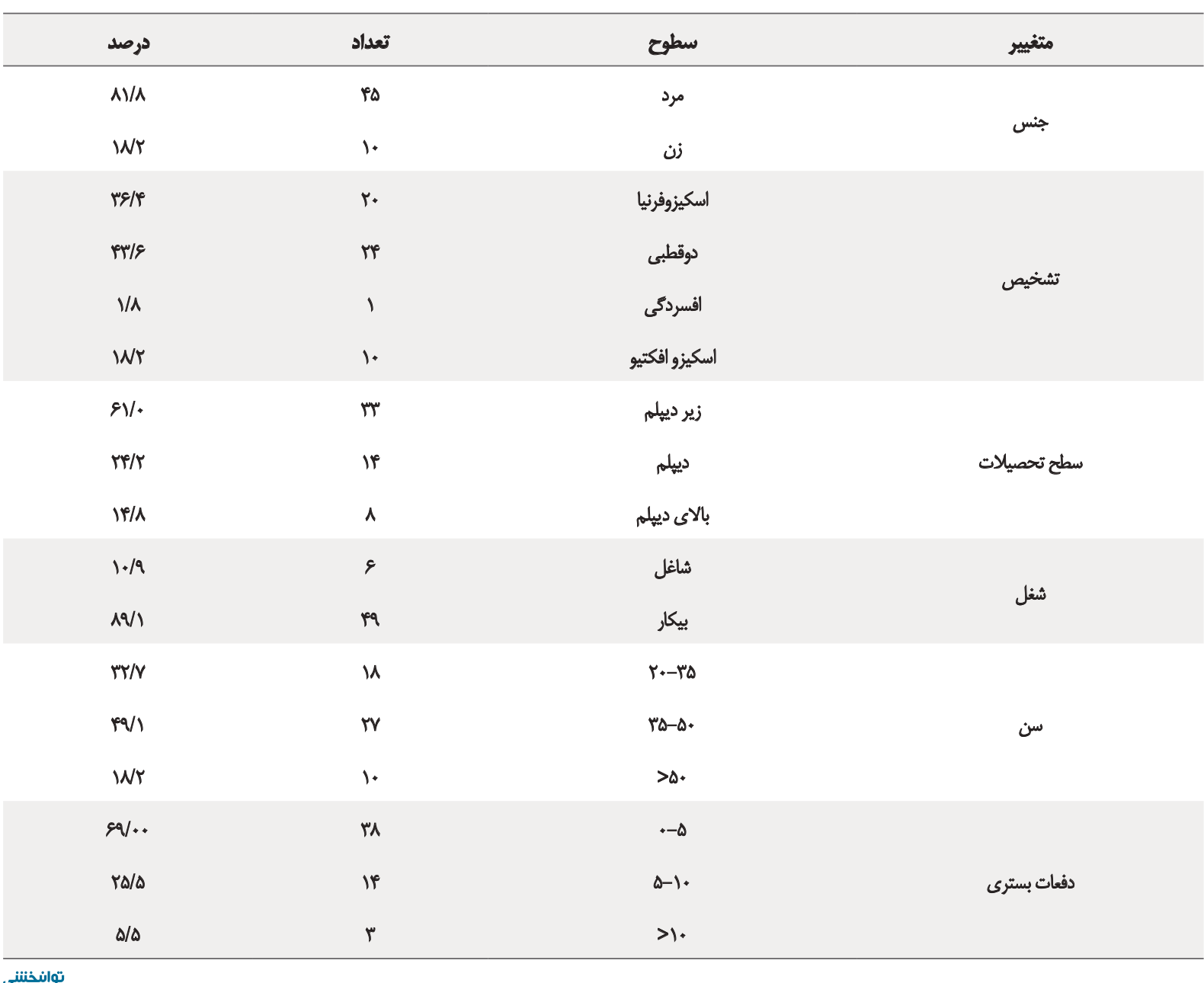

توانبخننى 
جدول ب. ميانكينه انحرافمعيار و ضريب آلفاي كروئباخ اجزاى آزمون بررسى عملكرد كاركردى بيىإِيا - ارزيابى مبتنى بر تكليف

\begin{tabular}{|c|c|c|c|c|c|}
\hline ألفاى كرونباخ & انحرافمعيار & مياتكين & بيشترين & كمترين & حوزه \\
\hline . /As & $r / M$ & $I r / 9 F$ & 19 & $\Delta$ & حافظة \\
\hline . /AF & $4 / .9$ & $|W / A|$ & r. & $\Delta$ & سازمائدهى زعان و وسايل \\
\hline .19 & 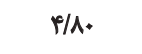 & $1 Q / \mathrm{V}$ & r. & $\Delta$ & محلودهة توجه \\
\hline .119 & P/Me & $\mid r / * \Delta$ & r. & $\Delta$ & الختنالال در تفكر \\
\hline .119 & f/at & $\mid W / \bullet \Delta$ & r. & $\Delta$ & توانايي تفكر انتزاعى \\
\hline . /AS & $1 / 19$ & $\mid r / 4$. & r. & $\Delta$ & تكميل تكليف \\
\hline$\cdot M$ & $H(\Delta)$ & Nq & 19 & $\Delta$ & خطاها \\
\hline 年 & T/AT & $N \notin 1$ & 10 & $\Delta$ & كارايع \\
\hline - /AF & $r / A I$ & $I T / Y$. & r. & $\Delta$ & انكيزش/انطبات \\
\hline$+/ A Y$ & reft & $I F / N$ & $r+$ & $\Delta$ & تحمل نالميدى \\
\hline . /AF & $P / \Delta$ & $18 / r 9$ & r. & $\Delta$ & اعثماد به نثس \\
\hline$\cdot 1 N$ & $T / M$ & $\mathbb{I T / P}$ & r. & $\Delta$ & عاطظفه عمومى \\
\hline ./9M & $r+1$ & $£ \Delta / \Delta A$ & $u$ & ro & شنالهُت \\
\hline - /AT & 1.118 & $r a / r$ & af & 10 & عملكرد \\
\hline.$/ M$ & $18 / 01$ & $\Delta T / A V$ & $w$ & r. & رقتار \\
\hline.$/ 91$ & NHI & $\pi / Q$ & PA & ir & دستهبئلى صدفها \\
\hline (9T & V/81 & re/rq & Po & ir & يول و بازاريايى \\
\hline Jar & 1.199 & $r g / M e$ & Fo & ir & طراحى خانه \\
\hline.$/ Q 8$ & $1 . / A f$ & TNYE & FA & ir & ساخت هكعب \\
\hline \multirow[t]{2}{*}{.$/ Q$} & I./AT & $\pi / \lambda)$ & PA & ir & طراحى شخص در حال حركت \\
\hline & $P \Delta / V$ & $\mid F q / M$ & rrq & g. & لمدهة كل \\
\hline
\end{tabular}

توانبخنتى

همجنين با توجه به نظر متخصصان مقدار شاخص روايى محتوا

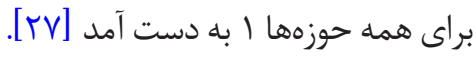

نتايج ضريب آلفاى كرونباخ r ا أتغير كاركردى، اجزا و ه تكليف

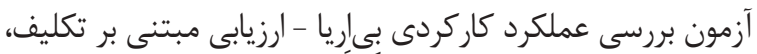

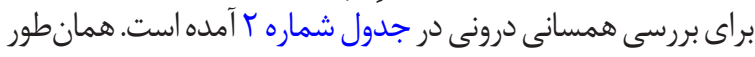

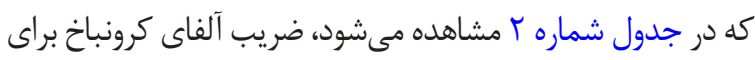

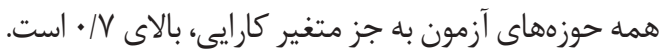

بحث

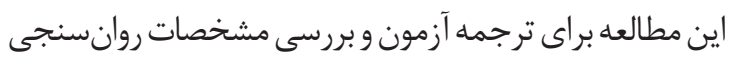

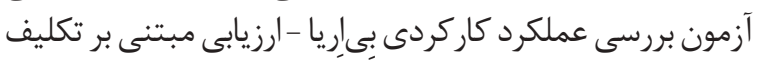

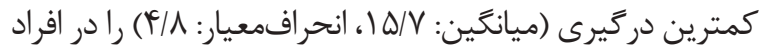
شركت كننده در مطالعه داشته است.

روايى صورى توسط متخصصان از نظر مطلوب بودن عبارات به إنهابه

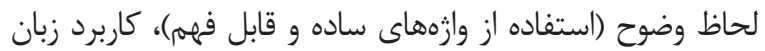

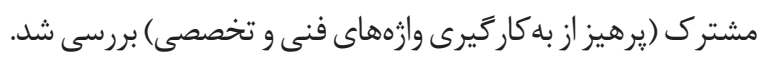

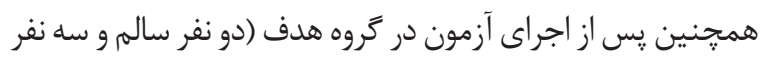

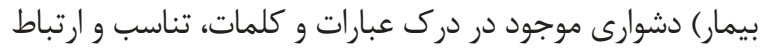

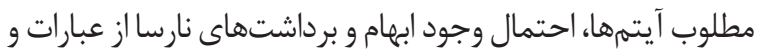

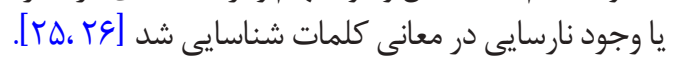

با توجه به نظر ينج متخصص در زمينه سلامت روان، مقدار شاخص نسبى روايى محتوايى براى همه حوز هها ا به دست دست آمد. 


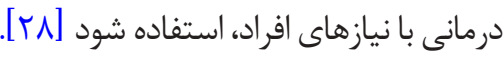
با توجه به هدف و محدوديت زمانى إى كه وجود داشت، تنها

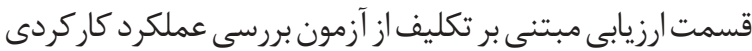

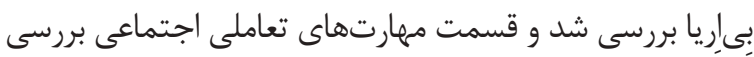

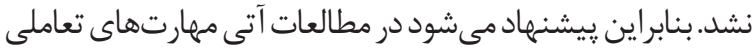

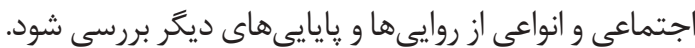

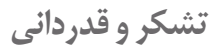

يزروهش حاضر از يايانامه كارشناسى ارشد نويسنده اول در

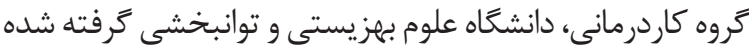

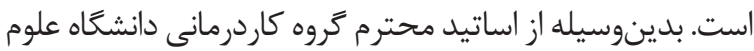

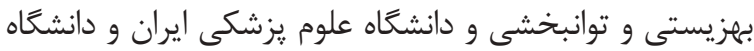

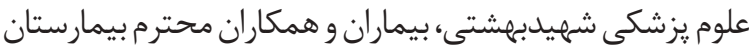

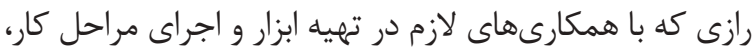

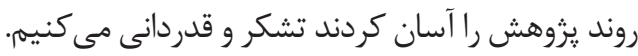

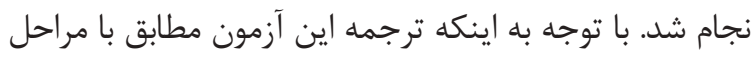

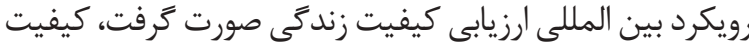

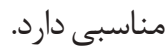

روايى صورى آزمون با توجه به نظر متخصصان و اجراى آن

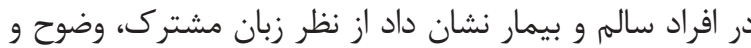

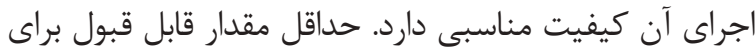

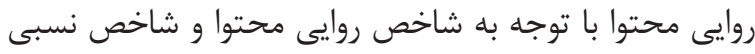

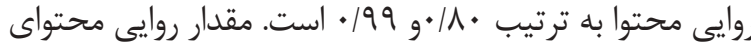

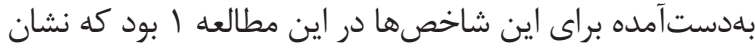
مى دهد نسخه فارسى اين ابزار، روايى محتواى مناسبى دارئ دارد. در مطالعهاى مشابه كه هوستون، ويليام، بلومر و من انجام

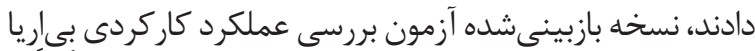

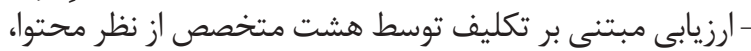

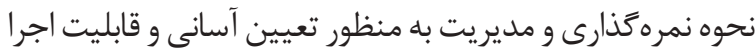

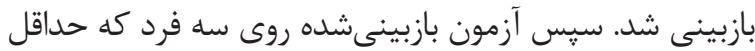

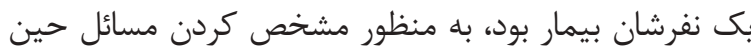

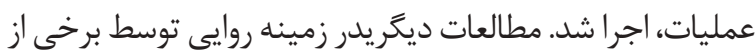

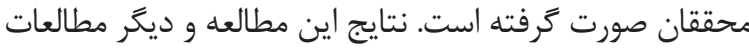

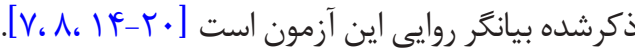
همسانى درونى آزمون با توجه به آلفاى كرونباخ بهدست آمده

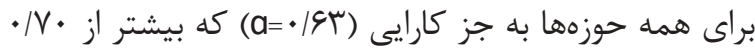

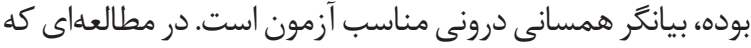

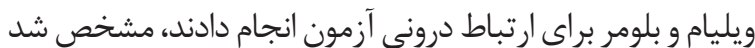

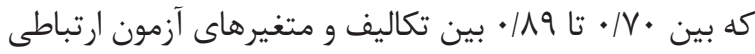

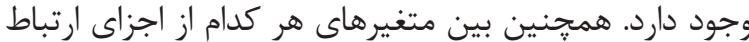

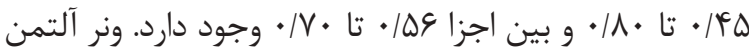

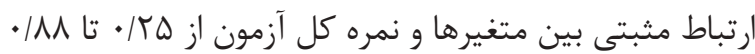

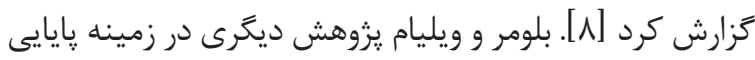

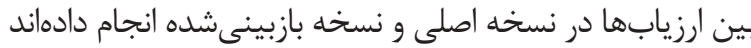

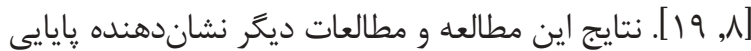

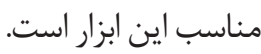

با توجه به جدول شماره r، ميانكَين و انحرافمعيار اجزاى

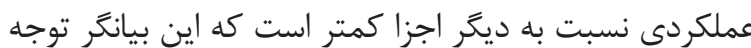

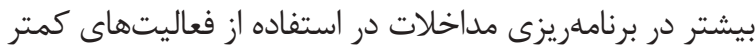

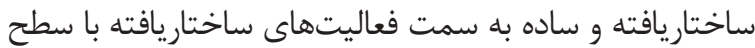
دشوارى مناسب است.

\section{تيجيَكيرى}

تتايج اين مطالعه نشان مىدهد اين ابزار قابليت استفاده در

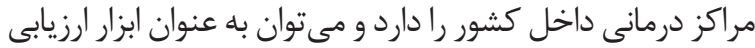

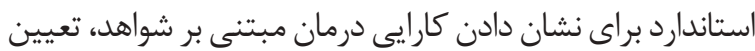
نقاط ضعف و قوت، برنامهريزى اهداف درمانى، و تناسب خدان خدمات 


\section{References}

[1] American Psychological Association. Diagnostic and statistical manual of mental disorders (DSM-5®). Washington D.C.: American Psychiatric Pub; 2013.

[2] Sharifi V, Hajebi A, Radgoodarzi R, Hefazi M. Twelve-month prevalence and correlates of psychiatric disorders in Iran: the Iranian Mental Health Survey, 2011. Archives of Iranian Medicine. 2015; 18(2):76 -84. [DOI:015182/AIM.004]

[3] Cara E, MacRae A. Psychosocial occupational therapy: An evolving practice. Toronto: Nelson Education; 2012.

[4] Atchison B, Dirette DK. Conditions in occupational therapy: Effect on occupational performance. Philadephia: Lippincott Williams \& Wilkins; 2007.

[5] Karbalaei Nouri A, Sadeghi A, Shamsolma'aali Z. [Construct validity confirmation of Iranian version of Lowenstein Occupational Therapy Cognitive Assessment (LOTCA) (Persian)]. Archives of Rehabilitation. 2009; 10(2):33-7.

[6] Fadai F. Rehabilitation of schizophrenia: At the end or in the beginning. Iranian Rehabilitation Journal. 2007; 5(1):53-5.

[7] Castilla LM, Klyczek JP. Comparison of the kinetic person drawing task of the Bay Area functional performance evaluation with measures of functional performance. Occupational Therapy in Mental Health. 1993; 12(2):27-38. [DOI:10.1300/ J004v12n02_02]

[8] Houston D, Williams SL, Bloomer J, Mann WC. The Bay Area functional performance evaluation: Development and standardization. American Journal of Occupational Therapy. 1989; 43(3):170-83. [DOI:10.5014/ajot.43.3.170]

[9] Roley SS, Barrows CJ, Susan Brownrigg OTR L, Sava DI, Vibeke Talley OTR L, Kristi Voelkerding BS, et al. Occupational therapy practice framework: Domain \& process. American Journal of Occupational Therapy. 2008; 62(6):625-83. [PMID: 19024744] [DOI:10.5014/ajot.62.6.625]

[10] Hojjati Abed E, Karbalaaei Nouri A, Rafiei H, Karimlou M. [The efficacy of psychosocial occupational therapy services on quality of life of chronic psychiatric patents (Persian)]. Archive of Rehabilitation. 2010; 11(1):23-8.

[11] Karbalaie Noori A, Hosseini SA. [Effect of Community Reentry Program (CRM) in patients with schizophrenia (Persian)]. Archives of Rehabilitation. 2013; 14(2):22-8.

[12] Karbalaee-Nouri A, Hosseini A, Hajebi A, Rafii H, Motevalian A. Independent and social living skills training for people with schizophrenia in Iran: A randomized controlled trial. Iranian Rehabilitation Journal. 2015; 13(3):84-88.

[13] Oakley F, Kielhofner G, Barris R. An occupational therapy approach to assessing psychiatric patients' adaptive functioning. American Journal of Occupational Therapy. 1985; 39(3):147-54. [DOI:10.5014/ajot.39.3.147]

[14] Accardi MA. The Bay Area functional performance evaluation: a validity study [PhD dissertation]. Medford: Tufts University; 1985.
[15] Bloomer JS, Williams SK. Bay Area functional performance evaluation: Behavioral guidelines. Palo Alto, CA: Consulting Psychologists Press; 1978.

[16] Thibeault R, Blackmer E. Validating a test of functional performance with psychiatric patients. American Journal of Occupational Therapy. 1987; 41(8):515-21. [DOI:10.5014/ajot.41.8.515]

[17] Klyczek JP, Mann WC. Concurrent validity of the task-oriented assessment component of the Bay Area functional performance evaluation with the American association on mental deficiency adaptive behavior scale. American Journal of Occupational Therapy. 1990; 44(10):907-12. [DOI:10.5014/ajot.44.10.907]

[18] Curtin M, Klyczek JP. Comparison of BaFPE-TOA scores for inpatients and outpatients. Occupational Therapy in Mental Health. 1993; 12(1):61-75. [DOI:10.1300/J004v12n01_05]

[19] Hemphill-Pearson BJ. Assessments in occupational therapy mental health: An integrative approach. Thorofare, NJ: Slack Incorporated; 2008.

[20] Thomson LK. The kohlman evaluation of living skills. Montgomery: American Occupational Therapy Association; 1992.

[21] Nobakht Z, Sourtigi H, Rassafiani M. [Translation, cultural adaptation, validity and reliability of a test: Factors influencing researcher decisions (Persian)]. Scientific Journal of Rehabilitation Medicine. 2015; 4(4):173-83.

[22] Bullinger M, Alonso J, Apolone G, Leplège A, Sullivan M, Wood-Dauphinee $S$, et al. Translating health status questionnaires and evaluating their quality. Journal of Clinical Epidemiology. 1998; 51(11):913-23. [DOI:10.1016/S0895-4356(98)00082-1]

[23] Nobakht Z, Rassafiani M, Reza Soltani P. Validity and reliability of Persian version of Craig Hospital Inventory of Environmental Factors (CHIEF) in children with cerebral palsy. Iranian Rehabilitation Journal. 2011; 9(1):3-10.

[24] Mohammad Beigi A, Mohammad Salehi N, Aligol M. [Validity and reliability of the instruments and types of measurments in health applied researches (Persian)]. Journal of Rafsenjan University of Medical Sciences. 2015; 13(12):1153-70

[25] Taylor RR. Kielhofner's research in occupational therapy: Methods of inquiry for enhancing practice. Philadelphia: FA Davis; 2017.

[26] Schell BA, Gillen G, Scaffa M, Cohn ES. Willard and spackman's occupational therapy. Philadelphia: Lippincott Williams \& Wilkins; 2013.

[27] Kamalian Lari S, Haghgoo HA, Farzad M, Hosseinzadeh S. [Investigation of the validity and reliability of Balance Evaluation Systems Test (BESTest) in assessment of balance disorders in people with multiple sclerosis (Persian)]. Archives of Rehabilitation. 2018; 18(4):288-95. [DOI:10.21859/jrehab.18.4.3]

[28] Managh MF, Cook JV. The use of standardized assessment in occupational therapy: The BaFPE-R as an example. American Journal of Occupational Therapy. 1993; 47(10):877-84. [DOI:10.5014/ajot.47.10.877] 